\title{
Exploring the Limits of Leakage Power Reduction in Caches
}

\author{
YAN MENG, TIMOTHY SHERWOOD and RYAN KASTNER \\ University of California, Santa Barbara
}

If current technology scaling trends hold, leakage power dissipation will soon become the dominant
source of power consumption. Caches, because of the fact that they account for the largest fraction
of on-chip transistors in most modern processors, are a primary candidate for attacking the leakage
problem. While there has been a flurry of research in this area over the last several years, a major
question remains unanswered-What is the total potential of existing architectural and circuit
techniques to address this important design concern? In this paper, we explore the limits in which
existing circuit and architecture technologies may address this growing problem. We first formally
propose a parameterized model that can determine the optimal leakage savings based on the perfect
knowledge of the address trace. By carefully applying the sleep and drowsy modes, we find that
the total leakage power from the L1 instruction cache, data cache, and a unified L2 cache may be
reduced to mere $3.6,0.9$, and $2.3 \%$, respectively, of the unoptimized case. We further study how
such a model can be extended to obtain the optimal leakage power savings for different cache
configurations.

Categories and Subject Descriptors: B.3.2 [Memory Structures]: Design Styles-Cache memories; C.0 [Computer Systems Organizations-General]: Modeling of Computer Architecture

General Terms: Algorithms, Experimentation, Performance

Additional Key Words and Phrases: Limits, cache intervals, leakage power

\section{INTRODUCTION}

Power dissipation has become a major concern to those designing processors for high-performance desktops, servers, and battery-operated portable devices. Higher energy dissipation requires more expensive packaging and cooling technology, which, in turn, increases cost and decreases system reliability. There are fundamentally two ways in which power can be dissipated: either dynamically (due to the switching activity of repeated capacitance charge and discharge on

Authors' addresses: Y. Meng, R. Kastner, Department of Electrical and Computer Engineering, University of California, Santa Barbara, Santa Barbara, CA 93106-9560; emails: yanmeng@engr.ucsb.edu; kastner@ece.ucsb.edu; T. Sherwood, Department of Computer Science, University of California, Santa Barbara, Santa Barbara, CA 93106-9560; email: sherwood@ cs.ucsb.edu

Permission to make digital or hard copies of part or all of this work for personal or classroom use is granted without fee provided that copies are not made or distributed for profit or direct commercial advantage and that copies show this notice on the first page or initial screen of a display along with the full citation. Copyrights for components of this work owned by others than ACM must be honored. Abstracting with credit is permitted. To copy otherwise, to republish, to post on servers, to redistribute to lists, or to use any component of this work in other works requires prior specific permission and/or a fee. Permissions may be requested from Publications Dept., ACM, Inc., 1515 Broadway, New York, NY 10036 USA, fax: +1 (212) 869-0481, or permissions@acm.org.

(C) 2005 ACM 1544-3566/05/0900-0221 $\$ 5.00$ 


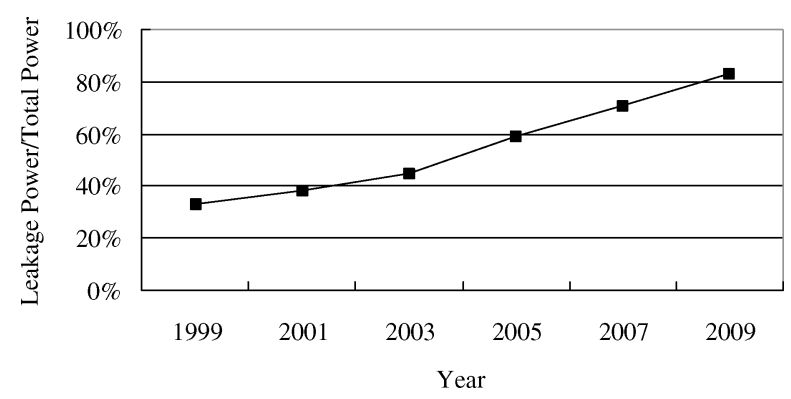

Fig. 1. Projected leakage power consumption as a fraction of the total power consumption according to the International Technology Roadmap for Semiconductors [ITRS].

the output of the millions of gates), or statically (mainly due to subthreshold and gate leakage [Kim et al. 2003; Rabaey et al. 2002]). Dynamic power consumption is proportional to the square of the supply voltage, which reduces as process technology scales. While the scaling down of transistor geometries enables reduction of the dynamic power, it greatly worsens the leakage problem. If current technology scaling trends hold [International Technology Roadmap for Semiconductors (ITRS)], leakage will soon become the dominant source of power consumption and, as such, new techniques are needed to battle this growing problem.

The problem of leakage becomes more significant as threshold voltage, channel length, and gate-oxide thickness are reduced. Furthermore, the subthreshold leakage, as a major component, stems from the need for a trade-off between dynamic power and performance. Scaling down the transistor supply voltage reduces the dynamic power dissipation. Yet, to maintain high switching speed under reduced voltages, the threshold voltage must also be scaled. As the threshold voltage drops, it is easier for current to leak through the transistor resulting in significant leakage power dissipation. The increases in device speed and chip density exacerbate the leakage problem. New technologies targeted at reducing dynamic power and increasing performance, such as low-threshold voltage [Liu and Svensson 1993] and gate-oxide scaling [Lee et al. 2004], further increase the relative importance of leakage power [ITRS] (Figure 1).

Cache memories have long been used to reduce the ever-growing gap between processors and memory. Modern processors typically provide two levels of onchip caches (e.g., separate L1 instruction and data caches and a unified L2 cache). In these processors, a large and growing fraction of the total on-chip area, and an even larger fraction of the total number of transistors, is consumed by caches. Because they account for such a significant portion of the total chip real estate, caches provide a healthy-sized target for designers to try circuit and architectural optimizations with the goal of reducing leakage power. The central idea behind most of these techniques is to exploit some form of temporal locality. By putting infrequently or unused cache lines into low leakage mode, much of the power will be reduced. By keeping frequently accessed cache lines active, total performance will not be reduced significantly.

Although there are several circuit techniques and management schemes concerning how and when to turn on or off individual cache lines, little work has 
been done to explore the limit of how well such techniques can work. What is the best we could hope to do with a given low-power technology? The primary goal of this paper is to explore these limits under different architectural and design assumptions in the hope of guiding research effort on leakage power in much the way that Belady's OPT algorithm [Belady 1966] helps (and continues to help) in the study of replacement policies.

There has already been much work on leakage power reduction and any proposed methods for calculating the limits of their effectiveness must be both general enough to capture a variety of techniques, yet specific enough to provide useful bounds. Our methods capture both state-preserving and destroying techniques and, in addition, we show how to optimally combine two such techniques into a hybrid scheme. We show that, given perfect knowledge of the future address trace, there exists a break-even point between drowsy and gated- $V_{d d}$. If the same cache line is accessed twice in an interval of time less than or equal to this break-even point, then drowsy mode should be used. If the same cache line is not used again within an amount of time greater than the break-even point, then more power can be saved by turning off the cache line using gated- $V_{d d}$. If these timings are known, then an optimal policy can be achieved.

Clearly perfect knowledge of the future trace is not always known, but it serves several purposes. First, it provides an important bound. No management method will be able to beat our power-reduction scheme under the given circuit assumptions. Second, it demonstrates that there is still a great deal of potential for policy decisions (when to turn a cache line on or off) to significantly reduce leakage power. Finally, while perfect knowledge of future references cannot be known, it can ofttimes be approximated by architecture techniques such as address prediction or prefetching [Meng et al. 2005].

In particular, we make the following contributions:

- We relate the potential savings that can be obtained from drowsy and gated$V_{d d}$ techniques under various assumptions for both the L1 instruction and data caches, and the unified L2 cache.

- We show that with oracle knowledge of future accesses, a simple optimal power-management scheme can be derived from a small set of circuit parameters.

- In addition to showing the optimal leakage savings on a set of implementation parameters, we develop a parameterized model to determine the optimal leakage savings, while the implementation technologies and architectures change over time.

- We show that while both drowsy and gated- $V_{d d}$ schemes are useful on their own, when combined, we can push the upper bounds of the leakage power savings to $96.4,99.1$, and $97.7 \%$ for the instruction cache, the data cache, and the unified L2 cache, respectively, with the 70-nm implementation technology.

- We also show that the model can be applicable to explore the limits of leakage power savings for different implementation technologies and cache configurations. 
- In addition to the limits study for the L1 instruction and data caches, we study the limits for L2 caches when both sleep and drowsy modes are employed. L2 caches, which take a large amount of area in modern processors, provide another interesting target to battle the leakage problem. Without careful attention to power, L2 caches may overtake the chip's power budget.

- We conduct the leakage study on different cache configurations to validate our methods.

- Instead of examining the leakage reduction on only five benchmarks [Meng et al. 2005], in this study we investigate our methods across all the SPEC2000 benchmark applications.

- We also empirically study the interval distribution to show the percentage that short dead intervals contributes to the total leakage reduction.

The rest of the paper is organized as follows. We review related work and motivate our limit study in Section 2. In Section 3, we propose our method for combining the gated- $V_{d d}$ and the drowsy method. We also explore the limit of leakage power saving that we can potentially achieve using our hybrid scheme. A model which parameterizes all the individual assumptions is also proposed. Section 4 describes our simulation setup and the benchmarks in our study and shows the results of our empirical study in exploring the upper bounds. We also study the generality of the parameterized model on different cache configurations. We offer concluding remarks in Section 5.

\section{CIRCUITS AND ARCHITECTURES OF REDUCED CACHE LEAKAGE POWER}

In order to derive a useful limit for leakage power reduction in caches, we must first begin with a discussion of those related technologies so that our model will be grounded in reality. In this section, we review several circuit techniques and develop the general ideas of our approach.

Leakage power comes from transistors that are simply left on. The easiest way to think about reducing the amount of the consumed leakage power is to "turn off" transistors that are not needed. While this is the easiest to think about, it is by no means the easiest to implement. One such approach, gated- $V_{d d}$ [Powell et al. 2001], attempts to solve this problem by reducing leakage through the use of a high-threshold sleep transistor (between pull-down NMOS and virtual $V_{s s}$ ) to break the connection, thus increasing the L1 cache line access time. This transistor is in the read critical path, which may impact performance. However, we only consider the potential for energy savings in this paper. This leakage-reduction technique is often called sleep mode and this is the naming convention that we use here. While efficient in saving leakage, sleep mode does not preserve the state of the data. When a cache line is needed again after it has been put to sleep, it must be refetched from lower levels of the memory hierarchy. This refetch is essentially an extra cache miss and this process can take many cycles, depending on the memory hierarchy, architectural assumptions, etc.

A different way of saving leakage power in the caches is to make use of multiple supply voltages. When the cache line is left fully on, it will dissipate 
too much leakage power. If $V_{d d}$ is fully gated, it will use very little power, but the data is lost. A compromise is to use a lower supply voltage when data is not needed for a while. This will reduce the leakage power without losing the data. The tradeoff is that while data will be preserved at this low-supply voltage, it cannot be accessed while in this state. Thus, there is a small wakeup time associated with changing from the lower voltage up to $V_{d d}$ (hence the name "drowsy"). If this can be implemented without adding a high-Vth transistor in the read critical path as the initial proposal, drowsy mode has the potential to achieve a smaller L1 cache access time than sleep mode. Drowsy mode does not fully turn off the memory, and thus does not reduce the leakage power as much as gated- $V_{d d}$. For a piece of data that is not going to be accessed for a very long time, sleep mode will be better because it reduces more leakage power. For a piece of data that is accessed in a moderate amount of time, drowsy mode will be better because there is not a large refetch penalty. This sets up one of the fundamental questions answered in our paper-how long is long enough for each mode?

While our paper attempts to address a previously unanswered question, there is a great deal of prior work aimed at reducing leakage power in caches. Azizi et al. [2003] introduced asymmetric dual- $V_{t}$ SRAM cell caches(ACCs), which exploit the fact that in ordinary programs most of the bits in caches are zeros for both the data and instruction streams and provide significant leakagereduction in the zero state. DRI-cache [Powell et al. 2001] uses the gated- $V_{d d}$ technique to dynamically adjust the size of the active portion of the cache by turning off a bank of cache lines based on the miss rates. DRG-cache [Agarwal et al. 2002] employs gated- $V_{d d}$ to reduce leakage power by turning off the gatedground transistor, while data is restored when the gated-ground transistor is turned on. DTSRAM [H. Kim and Roy 2002] uses body biasing to separately control the $V_{t}$ of each cache line. To minimize the energy and delay overhead, a cache line is switched to high $V_{t}$ when it is not likely to be used anymore. Kaxiras et al. [Hu et al. 2002; Kaxiras et al. 2001] proposed the cache-line decay scheme to turn off the cache lines in the dead periods of their cache generations using the gated- $V_{d d}$ technique. Instead of placing both the tag and the data into the sleep mode, AMC [Zhou et al. 2003] keeps the tag alive and tracks the miss rate with respect to the ideal miss rate. This helps to dynamically adjust the turn-off interval and control the overall performance. Velusamy et al. [2002] used formal feedback-control theory to adaptively adjust the cache-decay interval and cache lines are turned off accordingly. Another approach to reducing leakage power is called drowsy cache [Flautner et al. 2002; Kim et al. 2002, 2004], which decreases the supply voltage of idle cache lines. Specifically, all cache lines are periodically placed into drowsy mode. Kim and Mudge [2004] studied techniques for data retention with lower supply voltage. Hu et al. [2003] employed drowsy cache to exploit program hot spots and code sequentiality for instruction cache-leakage management. Parikh et al. [Li et al. 2004] compared gated$V_{d d}$ and drowsy cache at different L2 latencies with HotLeakage and showed that gated- $V_{d d}$ is superior for a set of faster L2 latencies. Heo [2002] reduced bitline leakage by leaving bitlines open whose cache banks are not accessed. Hanson [2001] found that for L1 caches, MTCMOS, which is a state-preserving 
technique that operates multiple threshold voltages, outperforms gated- $V_{d d}$. In $\mathrm{Li}$ et al. [2003], the authors presented several architectural techniques that exploit the data duplication across the different levels of cache hierarchy. They found that the best strategy in terms of energy and energy-delay product is to place the L2 subblock into a state-preserving mode as soon as its contents are moved to L1 and to reactive it only when it is accessed. Bai et al. [2005] investigated the impact of $T_{o x}$ and $V_{t h}$ on power performance tradeoffs for on-chip caches. In contrast, Sankaranarayanan and Skadron [2004] and Zhang et al. [2002] studied software approaches. Sankaranarayanan and Skadron [2004] decided the decay interval through profiling and showed that the optimal decay intervals can be estimated with a reasonable degree of accuracy using this method. Zhang et al. [2002] studied using a compiler to control leakage energy by inserting power mode instructions that control the voltage for the cache lines.

All of the above approaches strive to develop a scheme for predicting when a section of the cache should be put into a low-power mode. They use some heuristics based on either static analysis or run-time behavior to determine what mode each line should be in. One major open question is: what is the best that these approaches could hope to do? Clearly some of the cache lines will have to be left in a high $V_{d d}$ mode so they can be accessed, but how many and for how long? Are these approaches the ultimate in policy leakage power reduction or is there still room for improvement?

\section{CALCULATING THE LIMITS OF LEAKAGE POWER-REDUCTION TECHNIQUES}

Now that we have reviewed the circuit and architecture techniques employed to reduce leakage power, we describe how to calculate the savings that could be achieved by an optimal approach.

\subsection{Cache Intervals}

Our analysis of the leakage power-saving limit relies on the idea of breaking up the life time of each cache line into a series of intervals. An interval is the time that a cache line rests between two accesses. If an interval is very long then it would be beneficial to put that cache line in sleep mode for the duration of that interval. If an interval is very short, it should be simply left in a high- $V_{d d}$ mode. If an interval is somewhere in the middle, perhaps drowsy mode would be the best.

To illustrate the above situations, let's take a two-level loop example (Figure 2) extracted from a human resource-management application. It counts the total number of people employed during a year. In the example, the interval $\left(I_{a d d}\right)$ of the two consecutive accesses to the same instruction add depends on the size of the inner loop. When the range of the inner loop variable $j$ is large, the interval $I_{a d d}$ is long, which indicates the cache line of add instruction should be put into sleep mode to save leakage power. When the range is very small, the interval $I_{a d d}$ is small, which means this cache line should be left in the high- $V_{d d}$ mode for fast accesses. While the range is in the middle, the drowsy 


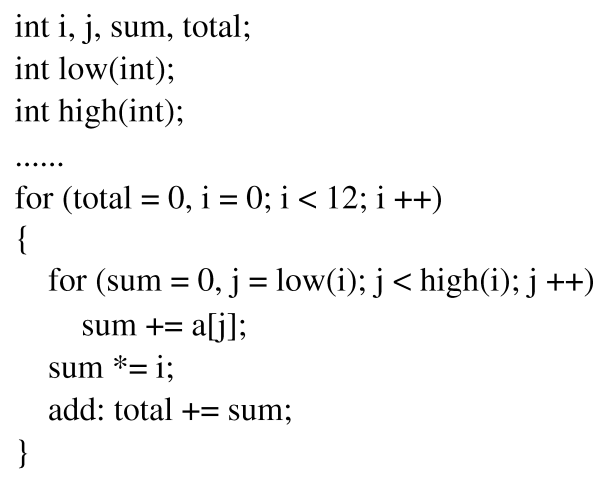

Fig. 2. The access interval example. The interval length of the consecutive accesses to the add instructions depends on the range of the inner loop $\mid$ high $(i)-l o w(i) \mid$.

mode should be applied to save leakage power without much performance cost. The idea behind our optimal scheme is to determine what the best policy would be for each interval in the program and then to apply the appropriate leakage technique to that interval.

In an optimal approach, each interval can be thought of as atomic in the eyes of the optimal policy. With oracle knowledge of the future address traces known (as would be for an optimal approach), there should be no reason to perform any new power-saving techniques in the middle of an interval. Instead, the same technique should have been applied for the entire duration of the interval as less power would be consumed with the same penalty (for either wakeup or refetch).

One thing to note is the notion of live intervals and intervals. A live interval starts when a new memory is brought into the cache frame and ends after the last access. Between the last access to a line of memory and the time it is evicted from the cache, it is regarded as dead. Besides turning off cache lines in dead periods as the cache decay scheme does [Kaxiras et al. 2001], our method also explores the live period of a cache generation, which demonstrates great potential for leakage-reduction. In fact, we found that dead periods did not contribute a large amount of leakage savings in the optimal case, because any long interval would be turned off whether live or dead. The only additional savings that are achieved from considering dead intervals are from short dead intervals, of which there are very few. Figure 3 is used to demonstrate such a point. It is drawn based on our experimental setup (see Section 4.1). The $x$-axis shows the interval length; it is $\log _{2}$-scaled. The $y$-axis shows the cumulative percentage of the live intervals over the sum of all live intervals and dead intervals over the sum of all dead intervals of the unified L2 cache for crafty and vortex. As it can be seen, the curves of the dead-interval-crafty and dead-interval-vortex arise when the interval lengths are large (greater than $2^{20}$ cycles); the short dead intervals only contribute an insignificant amount (less than 1\%) for the sum of all the dead intervals, which indicates that the short dead intervals contribute little to the leakage power reduction. Thus, for the rest of this paper, 


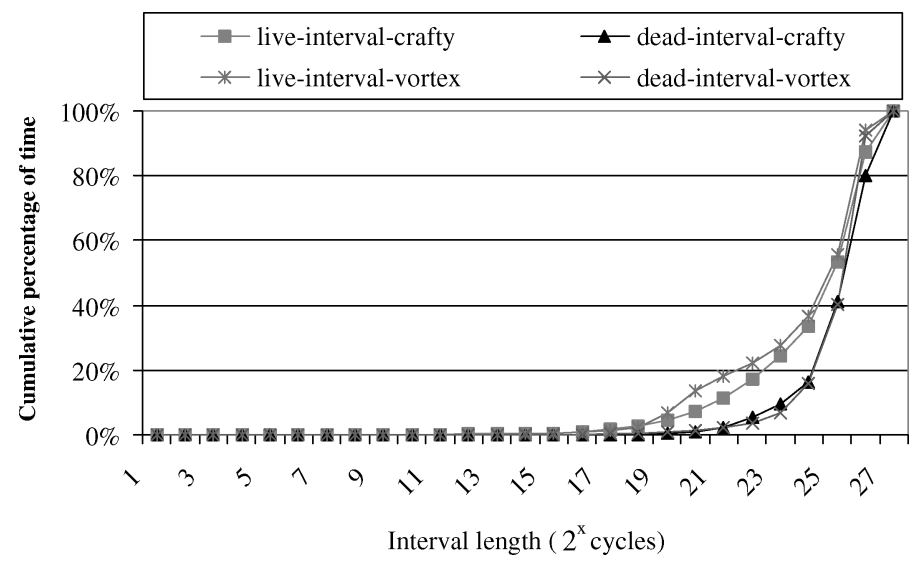

Fig. 3. Cumulative distribution of live intervals and dead intervals of the L2 cache for crafty and vortex. The total amount of short dead intervals only contribute little to the leakage power reduction, while the long intervals play a major role.

we ignore the effect of live and dead intervals and, instead, concentrate on only the durations of the intervals.

\subsection{Optimal Approach}

Our optimal approach works as follows. Given an interval distribution of cache accesses, which can be obtained based on a memory configuration, our optimal approach first classifies each cache access interval into one of the following three types: sleep-mode optimal, drowsy-mode optimal, and active-optimal, and applies the appropriate mode on each interval to obtain the optimal leakage power-saving. If the size of an interval is very small (i.e., there are multiple consecutive accesses within a short period of time), then it is best to leave the cache line in a fully active (nonpower saving) mode. If the size of an interval is long, then the best policy is to completely turn off that cache line (sleep) and then refetch it when it is needed again. The final case is if the interval size is neither very long nor very short. In this case it is best to put the cache line into a drowsy state, which consumes a small amount of power, has a small wakeup cost, and has the advantage of retaining the data values.

The key to dividing intervals into these categories is knowing the precise length of an interval that should be put into sleep mode, drowsy mode, or left active. The interval length where the power-saving mode changes is an inflection point, of which there are two: one between sleep and drowsy modes and the other between drowsy and active modes.

One thing to note is that our optimal approach will have no effect on the performance of the machine. Because we assume perfect access pattern knowledge, an optimal approach can refetch any needed data just before it is needed and avoid any impact on performance. By exploiting this fact, we can separate out the power problems from the performance problems. Although a just-in-time refetch or perfect prefetching will not affect the performance of the machine, it does have a power cost, which we consider later in this paper. Figure 4 is used to illustrate this point. In the sleep mode, due to turning off the cache 


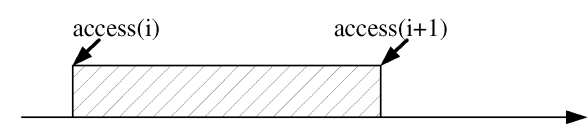

(a) The active mode

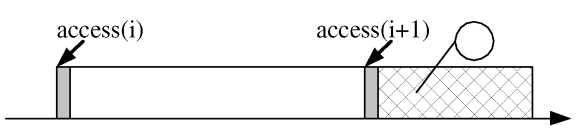

(b) The sleep mode w/o perfect prefetching

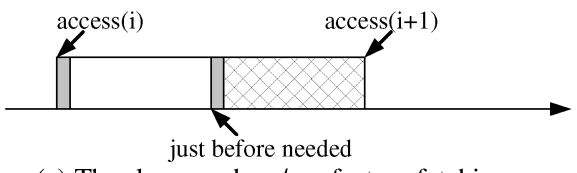

(c) The sleep mode w/ perfect prefetching

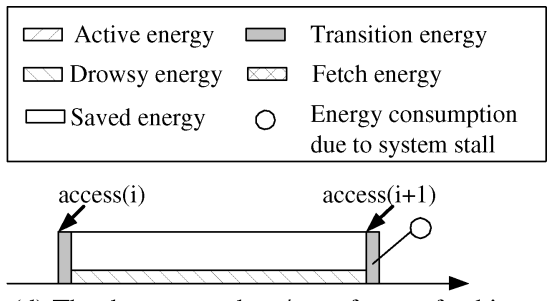

(d) The drowsy mode w/o perfect prefetching

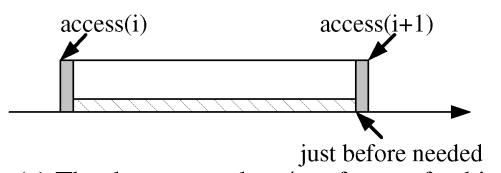

(e) The drowsy mode w/ perfect prefetching

Fig. 4. Using perfect prefetching to avoid performance degradation. Assuming perfect access pattern knowledge, an optimal approach uses perfect prefetching to refetch data just before it is needed and avoids stalling the whole system to reduce energy consumption.

line to save leakage power, the data is not preserve. If the data is accessed again, it needs to be refetched, and this refetching process may usually take several cycles. Without just-in-time refetch (Figure $4 \mathrm{~b}$ ), the other parts of the whole system will have to stall for that amount of cycles, waiting for the data to be ready. The stall will lead to significant energy consumption, as the large circle indicates. Similar things happen to the drowsy mode. This mode preserves the data and takes only a couple of cycles [Kim et al. 2004] to wake up the cache line. Without just-in-time refetch (Figure 4d), the amount of energy the drowsy mode consumes is less than that of the sleep mode during the system stalling, which is indicated by a small cycle. By contrast, with just-in-time refetch (Figure $4 \mathrm{c}$ and e), The data will be ready when it is needed, avoiding stalling the rest parts of the whole system to wait for data to be ready, which consequently saves power. It is worth noting that our scheme calculates the optimal powersavings for a given replacement policy, it does not change the replacement policy to further save power.

For the convenience of illustrating how our approach works in general, we will use Figure $5 \mathrm{a}$ and $\mathrm{b}$ to show how the inflection points are calculated. Figure 5a shows that the sleep mode and the drowsy mode require time to reduce the voltage from high $V_{d d}$ to off $\left(s_{1}\right)$ and from high to $V_{d d_{l o w}}\left(d_{1}\right)$, respectively. There is also a similar time overhead in coming out of the mode $\left(s_{3}\right.$ or $d_{3}$ ). For the sleep mode, since the latency $D$ of fetching data from L2 cache is longer than $s_{3}$, there is another overhead $\left(s_{4}=D-s_{3}\right)$ before the next access. We divided the lifetime of an interval into several durations to illustrate these overheads. Figure $5 \mathrm{~b}$ shows the length of each duration $s_{1}, s_{2}, s_{3}, s_{4}, d_{1}, d_{2}, d_{3}$ in an access interval of both a sleep mode and a drowsy mode. The total length of the cache access interval using the sleep technique is $s=s_{1}+s_{2}+s_{3}+s_{4}$, which that of using the drowsy mode is $d=d_{1}+d_{2}+d_{3}$.

For the sleep mode, the data has been lost due to an induced miss [Kaxiras et al. 2001] and must be refetched from the memory hierarchy. As such, there is a significant amount of power consumed by the dynamic activity required to 


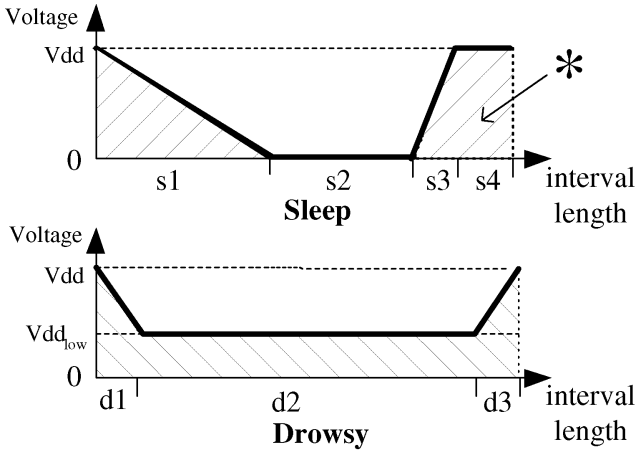

( a )

\begin{tabular}{|c|c|}
\hline Duration & Voltage \\
\hline $\mathrm{s} 1$ & High to off \\
\hline $\mathrm{s} 2$ & Always off \\
\hline $\mathrm{s} 3$ & Off to high \\
\hline $\mathrm{s} 4$ & High \\
\hline $\mathrm{d} 1$ & High to low \\
\hline $\mathrm{d} 2$ & Always low \\
\hline $\mathrm{d} 3$ & Low to high \\
\hline
\end{tabular}

* Dynamic power consumption due to an induced miss

( b )

Fig. 5. Time-voltage diagrams of sleep-mode and drowsy-mode. In Sleep-Mode the cache line is essentially turned completely off and the power consumed drops to nearly zero. While beneficial over a long period of time, there is a more significant overhead due to refetch. Drowsy-Mode has a smaller overhead, but the cache line still consumes a measurable amount of power because the voltage has not been completely turned off.

fetch the data from the L2 cache, [marked with an asterisk (*) in Figure 5b]. This dynamic power cost can be obtained from analytical models, such as the interconnect model based on logical effort [Amrutur and Horowitz 2001] or the CACTI [Shivakumar and Jouppi 2001] model, which has been used in this paper.

The sleep-drowsy inflection point is derived as the access interval length when the sleep and the drowsy modes consume the same amount of energy. If the interval is of a length less than the inflection point, then drowsy mode would be optimal. If it is greater than the inflection point then sleep mode would be optimal. We denote the leakage power consumption of each cache line as $P_{L}$, which can be obtained from the HotLeakage tool [Zhang et al. 2003] and the cost of dynamic power due to an induced miss for the sleep mode as $C_{D}$. The energy of a sleep-mode interval can be calculated as Eq. (1):

$$
E_{S}=\sum_{i=1}^{4} P_{L}\left(s_{i}\right) * s_{i}+C_{D}
$$

Similarly, the energy consumption using the drowsy model can be calculated as Eq. (2):

$$
E_{D}=\sum_{i=1}^{3} P_{L}\left(d_{i}\right) * d_{i}
$$

When the two modes consume the same amount of energy, Eq. (3) is reached:

$$
E_{S}=E_{D}
$$

Applying the data in Figure 5b into Eq. (3), we can calculate the sleep-drowsy inflection point.

The other inflection point is between drowsy and active modes. The drowsyactive inflection point is calculated as the sum of the durations $d_{1}$ and $d_{3}$, within which the voltage changes either from $V_{d d}$ to $V_{d d_{l o w}}$ or from $V_{d d_{l o w}}$ to $V_{d d}$. 


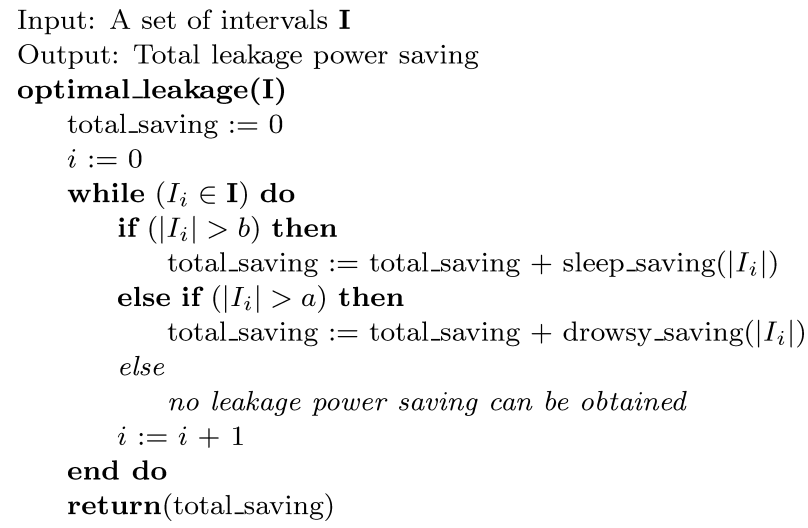

Fig. 6. Algorithm to compute the optimal leakage power saving given an interval distribution. Intervals are classified into one of the three categories based on the drowsy-active inflection point $a$ and the sleep-drowsy inflection point $b:(0, a],(a, b]$, and $(b,+\infty)$. The sleep-mode is applied on intervals within the range of $(b,+\infty)$; the drowsy mode is applied on intervals within the range of $(a, b]$; the cache lines are left on for intervals within the range of $(0, a]$.

Note that the sleep-drowsy inflection point is the point at which sleep mode has the potential to save power of drowsy mode. sleep mode does not provide benefit at small interval lengths because of the larger penalty associated with coming out of sleep mode (the power of refetch) as opposed to drowsy mode. The only way to save power on small-interval lengths is to know exactly when the cache line will be reaccessed so that it can be brought out of sleep mode before the data is needed. This is how an optimal leakage-management scheme would take advantage of perfect knowledge.

When an interval between two accesses to the same cache line is longer than the sleep-drowsy inflection point, using sleep mode has the potential to save more leakage power. When an interval is less than the sleep-drowsy inflection point but still greater than the active-drowsy inflection point, the drowsy mode saves more leakage. When its interval length is less than the active-drowsy inflection point, the cache line is always active and cannot have its leakage power reduced without causing a delay in delivering the data.

Figure 6 details our optimal leakage power-saving approach. By classifying cache intervals into the three types and applying to them the appropriate leakage saving mode, the maximal leakage power-saving can be obtained as the accumulation of the leakage saving over all access intervals, which provides us an upper bound for optimal leakage power-savings. It can be proved that, based on the perfect knowledge of the lengths of all intervals, the optimal leakage power saving can be achieved by applying the proper operating mode on each interval.

\subsection{Theorem of the Optimal Policy for Leakage Power Saving}

In this section, after defining the relevant terms in our study, we provide the theorem of the optimal policy for leakage power saving.

Definition 3.3.1. We define $\mathbf{I}=\left\{I_{i}\right\}$ as a set of intervals and the length of interval $I_{i}$ as $\left|I_{i}\right|\left[\left|I_{i}\right| \in(0,+\infty)\right]$. 
Definition 3.3.2. For each interval $I_{i} \in \mathbf{I}$, we define three possible operating modes $T_{j} \in \mathbf{T}$, where $\mathbf{T}=\left\{T_{1}=\right.$ active, $T_{2}=$ drows $y, T_{3}=$ sleep $\}$, and the leakage energy saving of the interval $I_{i}$ working in the mode of $T_{j}$ is defined as $E\left(I_{i}, T_{j}\right)$.

Definition 3.3.3. We define two inflection points, the active-drowsy inflection point $a$ and the sleep-drowsy inflection point $b$. The active-drowsy inflection point $a$ is defined as the sum of the durations within which the supply voltage changes either from high to low or from low to high, i.e., $a=d_{1}+d_{3}$. The sleep-drowsy inflection point $b$ is defined as the access interval length when the sleep and the drowsy modes consume the same amount of energy, i.e., $b=s_{1}+s_{2}+s_{3}+s_{4}=d_{1}+d_{2}+d_{3}$, where $s_{i} \geq 0, d_{j} \geq 0(i=1,2,3,4 ; j=1,2,3)$.

Lemma 3.3.4. The active-drowsy inflection point a is less than the sleepdrowsy inflection point $b$.

PRoof. Because loads have physical capacities, the discharging process takes less time for the voltage dropping from high to low than from high to off, i.e., $d_{1}<s_{1}$. Similarly, the charging process takes less time for increasing the voltage from low to high than from off to high, i.e., $d_{3}<s_{3}$. Since there is no overlapping time between $s_{i}$ and $s_{k}(i \neq k, i, k=1,2,3,4)$ and $s_{i} \geq 0(i=1,2,3,4)$, we can conclude that the sum of the durations $a=d_{1}+d_{3}<s_{1}+s_{3}$, and the sleep-drowsy inflection point $b=s_{1}+s_{2}+s_{3}+s_{4}$ is greater than $s_{1}+s_{3}$. Thus, $a$ is less than $b$. Our study, based on the 70-nm technology process, also justifies that $a$ (six cycles) is less than $b$ (1057 cycles) from the experimental perspective.

THEOREM 3.3.5. Under the context of the independent model, where access intervals of a cache block are independent from each other, we assume that for each interval $I_{i} \in \mathbf{I}$, one, and only one, of the three operating modes $T_{j} \in \mathbf{T}$ can be applied for reducing leakage energy consumption based on the following policy:

1. When the interval length $\left|I_{i}\right| \in(0, a]$, the active operating mode or nonpower saving mode is applied.

2. When the interval length $\left|I_{i}\right| \in(a, b]$, the drowsy mode is applied.

3. When the interval length $\left|I_{i}\right| \in(b,+\infty)$, the sleep-mode is applied.

Then the maximal leakage saving can be obtained as the combination of the power saving over all intervals $I_{i} \in \mathbf{I}$, which gives an upper bound for optimal leakage power saving.

PRoof. We prove the theorem by contradiction. We divide the whole range of the interval length $(0,+\infty)$ into three independent portions, based on the active-drowsy inflection point $a$ and the sleep-drowsy inflection point $b$, i.e., $(0, a] \cup(a, b] \cup(b,+\infty)$ (see Lemma 1 that $a<b)$. Suppose the energy saving $M$, based on the above assumptions, is not maximal, then there must be another energy saving $M^{\prime}$ that is greater than $M$, which indicates that there is at least one interval $I_{i}$ whose operating mode $T_{j}^{\prime}$ is different from $T_{j}$.

Figure 7 shows the function of interval versus energy consumption. In the figure, we can have the following derivations: 


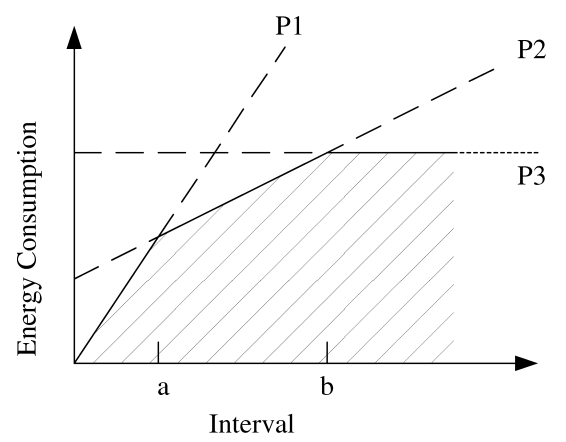

Fig. 7. Energy consumption for each of the three operating modes and the lower envelope $E\left(I_{i}, T_{j}\right)$ function for minimal energy consumption.

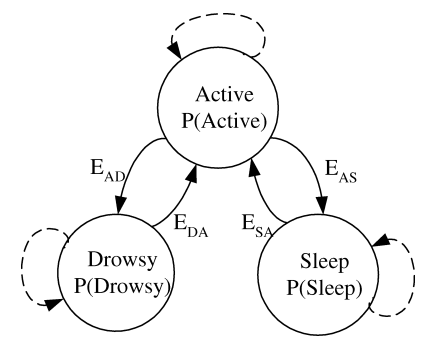

Fig. 8. The optimal leakage power-saving model. Circles indicate states; edges represent transitions between states.

1. The function is continuous and monotonically increasing.

2. The slopes $P_{1}, P_{2}$, and $P_{3}$ indicate the power consumptions within the interval ranges of $(0, a],(a, b]$, and $(b,+\infty)$, respectively.

3 . For intervals in the range of $(0, a]$, the minimal energy consumption can be achieved through the active mode $T_{1}$. For intervals in the range of $(a, b]$, the minimal energy consumption can be achieved through the drowsy mode $T_{2}$. For intervals in the range of $(b,+\infty)$, the minimal energy consumption can be achieved through the sleep mode $T_{3}$.

For a set of independent intervals, if at least one interval $I_{i}$ was applied with $T_{j}^{\prime}$, not the corresponding mode $T_{j}$, then $E\left(I_{i}, T_{j}^{\prime}\right)$ is greater than $E\left(I_{i}, T_{j}\right)$ (above the shadow area in Figure 7), giving the contradiction. Therefore, the maximal leakage power saving can be obtained by the proposed policy.

\subsection{Generalized Model for Optimal Leakage Power Savings}

After illustrating our optimal leakage power-saving approach, we evolve our approach to a complete model that can capture the optimal leakage savings as configurations and technologies change.

As Figure 8 depicts, the model has three states, which are indicated by the circles, representing the three operating modes: active, drowsy, and sleep. It also models the transitions between states, demonstrated by the edges (selfedges means that the state remains the same in the next cycle). Each state is 
associated with its static power consumption $(P)$, and the weights $\left(E_{A D}, E_{A D}\right.$, $\left.E_{A D}, E_{A D}\right)$ on the edges are the transition energy consumptions. For example, $E_{A D}$ is the energy consumption when transiting from the state active to the state drowsy.

In the model, all the individual assumptions, namely, the durations, energy costs of transitions between modes, the leakage power consumption of each mode, and the intervals, are parameterized and used as inputs to the model. The outputs of the model are the optimal leakage saving percentages of using the optimal sleep, optimal drowsy, and the optimal combining methods.

The model has been designed to explore the optimal leakage savings with parameterized architectural and design considerations, i.e., if the architectural configuration changes(for example, cache configurations), the model can adapt to the input change; if a new low-power mode is employed, the model can be easily extended by adding a new state.

The model for optimal leakage power-savings serves two major functions. First, instead of being an abstract model, it is coded in $\mathrm{C}$ language and is publicly available for cache-leakage studies. ${ }^{1}$ To use the tool, designers only need to feed the following inputs into the $\mathrm{C}$ program, such as the transition energies obtained from CACTI [Shivakumar and Jouppi 2001], the leakage power consumption from HotLeakage [Zhang et al. 2003], the interval distribution from SimpleScalar [Desikan et al. 2001], and the duration parameters $\left\{s_{1}, s_{2}\right.$, $\left.s_{3}, s_{4}\right\}$. The tool will then determine an optimal mode transition sequence that can achieve the maximal leakage power savings and output the optimal leakage saving percentages of using optimal sleep, optimal drowsy, and optimal combining methods. Second, and the most important, is that this model was designed to explore the optimal leakage savings under different architectural and design assumptions with the hope of guiding research effort on leakage power study.

Concurrent to our work, similar approaches are being developed to guide policy decisions in the domain of Computer Aided Design [Liu and Chou 2004]. In Liu and Chou [2004], the optimal energy mode transition sequence for generic devices is calculated under a fixed-delay constraint. Because of the complex timing involved in a modern superscalar microprocessor, a simple timing model will not accurately reflect the impact changing cache parameters. In our study on cache leakage, we have factored out any performance impact through perfect prefetching, which acts as a guiding bound for those developing leakagereduction schemes. We have built our model around the state of the art in leakage reduction techniques and show how the most common techniques considered today can be mapped onto this simple framework quickly and precisely. In addition, we have given a formal proof of optimality, which has not been offered in their work.

\section{EMPIRICAL STUDY}

In Section 3, we discussed the limits of leakage power-reduction techniques and how they are calculated. In this section, we show limit results gathered

${ }^{1} \mathrm{http}: / /$ express.ece.ucsb.edu/software/leakage.html.

ACM Transactions on Architecture and Code Optimization, Vol. 2, No. 3, September 2005. 
from actual benchmarks with parameters extracted from modern processors and prior work. Our objective is to evaluate the limits on some leakage powersaving techniques as applied to both the L1 instruction and data caches and the L2 cache. We show upper bounds on the possible savings using sleep mode, drowsy mode, or a potential hybrid of the two. We also evaluate the generality of the parameterized model in deriving the limits of leakage power-savings from the perspectives of different implementation technologies and different cache configurations.

\subsection{Methodology}

To test the amount of power that can be saved by using an improved leakage-reduction technique, we employed detailed cycle-level simulation. The simulator we use is a version of SimpleScalar closely resembling Compaq Alpha 21264 [Kessler 1999]. The execution core is a four-wide superscalar pipeline and the memory hierarchy includes a $64-\mathrm{KB}$, two-way set associative L1 instruction cache with a single-cycle hit latency, a 64-KB, two-way set associative L1 data cache with a three-cycle hit latency, and a unified 2-MB direct-mapped L2 cache with a seven-cycle hit latency. The main memory system consists of 16 32-MB DDR2 SDRAM chips [Samsung Electronics 2002], for a total main memory capacity of $512 \mathrm{MB}$, with an access time of $40 \mathrm{~ns}$, and an access power of $300 \mathrm{~mW}$. Because leakage is exponentially dependent on temperature, we use $85^{\circ} \mathrm{C}$ in our experiments. LRU is employed as the replacement policy throughout the memory hierarchy. To calculate inflection points, we assumed a $500-\mathrm{MHz}$ processor clock.

In order to capture the most important program behaviors, while at the same time reducing simulation time to reasonable levels, we used the simulation points that were described and verified in SimPoint [Sherwood et al. 2002]. The benchmark suite for this study consists of all the SPEC2000 benchmarks compiled for the Alpha AXP ISA. Because modern processors typically have two levels of on-chip caches (e.g., separate L1 instruction and data caches and a unified L2 cache), in the rest of our empirical study, we will first explore the limits of the leakage power savings for the L1 instruction and data caches. We will then conduct the limits study for the L2 caches.

\subsection{Limits Study for the L1 Instruction and Data Caches}

In this section, we explain how the optimal approach works from the experimental aspect in exploring limits of leakage power-savings for the modern L1 caches by the ways we calculate the inflection points and how we optimally combine the sleep and drowsy modes.

4.2.1 Calculating Inflection Points. Inflection points are the keys to choosing the best power saving mode of a given interval distribution. An intervals with its length greater than the sleep-drowsy inflection point is put into the sleep mode, and an interval with its length less than the active-drowsy inflection point is left in the active mode. For an interval with its length between the two inflection points it is put into drowsy mode to save power and has little performance impact. 
Table I. Active-Drowsy and Drowsy-Sleep Inflection Points Depicted in Cycles for Different Technologies

\begin{tabular}{|l|r|r|r|r|}
\hline Technology & $70 \mathrm{~nm}$ & $100 \mathrm{~nm}$ & $130 \mathrm{~nm}$ & $180 \mathrm{~nm}$ \\
\hline Active-drowsy point & 6 & 6 & 6 & 6 \\
\hline Drowsy-sleep point & 1057 & 5088 & 10328 & 103084 \\
\hline
\end{tabular}

To calculate inflection points with respect to different technologies, we used the durations $s_{1}=30, s_{3}=d_{1}=d_{3}=3$ and $s_{4}=4$ cycles [Li et al. 2004] ( $s_{2}$ and $d_{2}$ are dependent on an interval length). When we applied the parameters into Eqs. 1, 2, and 3, we obtained the inflection points for the L1 instruction and data caches shown in Table I. The table shows that the value of the sleepdrowsy point decreases while the technology scales down from 180 to $70 \mathrm{~nm}$ (These are the only currently available technologies provided by the Hotleakage [Zhang et al. 2003] tool. If, in the future, Hotleakage is extended to incorporate more technologies, our approach can still be applied to obtain their inflection points). This is due to the fact that the leakage power consumption per cache line increases while the dynamic energy consumption caused by an induced miss decreases with technology scaling down (see Eq. 3).

Since $70 \mathrm{~nm}$ is the most advanced technology that will be reached in a few years according to ITRS [International Technology Roadmap for Semiconductors], we employed it and its corresponding sleep-drowsy inflection point (1057 cycles) in the rest of our L1 caches study.

4.2.2 Combining Sleep and Drowsy Modes. With the inflection points calculated, the first question to be answered is how well a hybrid of sleep and drowsy modes can perform versus sleep mode. If, in the optimal case for sleep mode, we can perfectly predict the distances between access to cache lines, we can then potentially make use of sleep mode even if the cache line is accessed every 1057 cycles. In this case, there will be little benefit from using drowsy mode for those cache lines that are accessed more frequently than every 1057 cycles. However, if the threshold was different and if the inflection point between drowsy and sleep modes changed dramatically, there would be a point at which using both drowsy mode (for occasionally accessed line) and sleep mode (for rarely accessed lines) would become beneficial. The purpose of Figure 9 is to demonstrate this point. In our experiments, when a sleep mode is applied, the dynamic power consumption due to an induced miss was removed from the total leakage power savings.

The results in Figure 9a are derived based on the average leakage powersavings for both instruction and data caches across all given benchmarks. Through this figure, we examine the potential effectiveness of a pure sleep mode versus a hybrid sleep/drowsy method where we change the minimum interval length that can be put into sleep mode from 1057 to 10000 . These results indicate that a hybrid method (sleep + drowsy) can work consistently better than the sleep or the drowsy method alone, especially if one is very conservative about which lines are put to sleep. However, as the minimum sleep length approaches the sleep-drowsy inflection point (decreases), the usefulness of applying the drowsy method, in addition to the sleep mode, decreases. Under such conditions, the sleep mode removes most of the leakage power and thus 


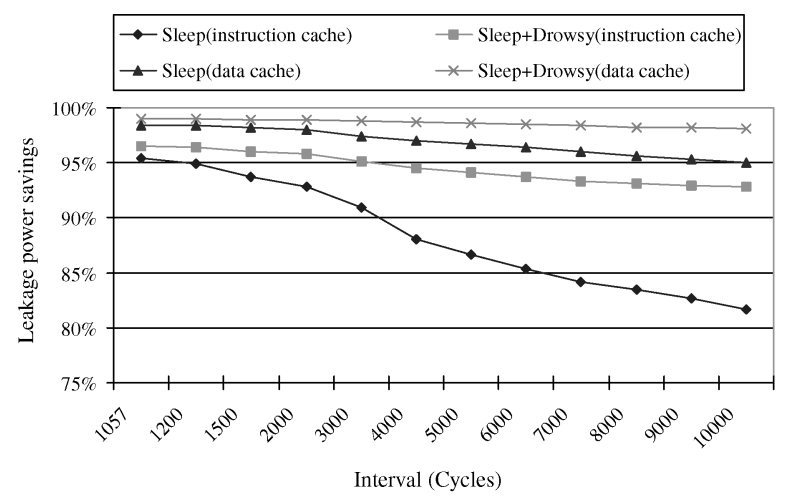

(a) L1 Instruction and Data Caches

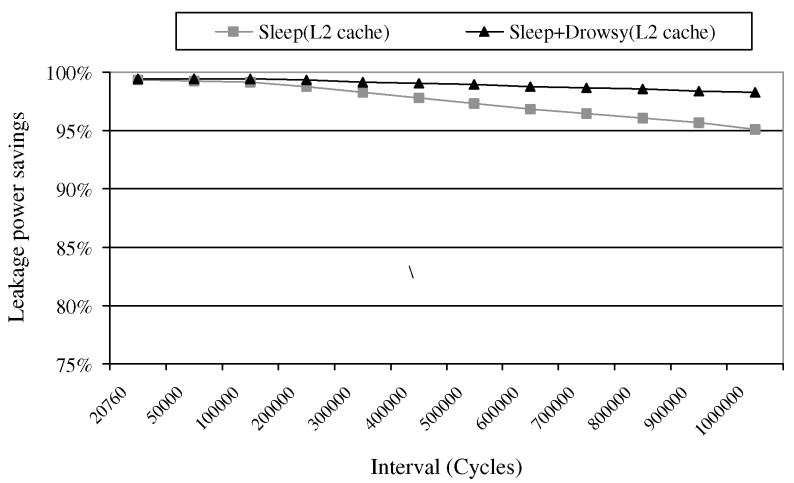

(b) Unified L2 Cache

Fig. 9. Comparison of the hybrid method versus the sleep-mode method for different sleep interval lengths. The usefulness of applying the drowsy method to save leakage power decreases as the sleep length approaches the sleep-drowsy inflection point. For leakage power saving, the sleep mode plays a more important role in the L2 cache and the data cache than in the instruction cache. The L2 cache has larger sleep intervals than the data cache.

there is not much more for drowsy to save. While clearly an implementable scheme will not have the luxury of perfect future knowledge, for those that we do have knowledge for, sleep mode should be applied very aggressively.

Moreover, the figure depicts that the gap between the hybrid method and the sleep mode for the data cache is much smaller than that for the instruction cache. The reason is that the same cache block in the data cache tends to be less frequently accessed than in the instruction cache and the interval lengths between consecutive accesses are much longer. Hence, the sleep mode plays a much more important role in the data cache for the leakage power saving than in the instruction cache.

Finally, this figure also confirms that the small variances of the sleep-drowsy inflection point will not significantly change our findings.

4.2.3 Exploring the Upper Bound. With the assumption of perfect prefetching, the upper bound of the leakage power saving was derived in 
Section 3, based on the two inflection points. We now explore the leakage powersaving limits of the following methods assuming perfect knowledge of the future address trace:

- OPT-Drowsy: An optimal drowsy cache that has no performance penalty for waking up data (although there is a power penalty, as discussed in Section 3).

- OPT-Sleep(10K): An optimal cache line-sleeping technique that puts to sleep all intervals of a size greater than $10 \mathrm{~K}$ with no performance penalty.

- Sleep(10k): ${ }^{2}$ Similar to the OPT-Sleep(10K) with the exception that instead of optimally turning off any cache line that has an interval larger than $10 \mathrm{~K}$, the line must now stay active for $10 \mathrm{~K}$ and then may be optimally slept.

- OPT-Hybrid: The method that optimally combines drowsy and sleep modes based on the inflection points without any performance penalty.

For the convenience of discussing the implementation of each technique, we define an access interval of a cache line as $T_{i}$. OPT-Drowsy puts the line into the drowsy mode during $T_{i}$, if $T_{i}$ is greater than 6 , while OPT-Sleep $(10 \mathrm{~K})$ puts the cache line into the sleep mode during $T_{i}$ if $T_{i}$ is greater than $10 \mathrm{~K}$. We also studied Sleep $(10 \mathrm{~K})$ to simulate the cache-decay scheme, whose decay interval is $10 \mathrm{~K}$. In this case, a cache line is put into the sleep mode for $\left(T_{i}-10 \mathrm{~K}\right)$ cycles if $T_{i}$ is greater than $10 \mathrm{~K}$. The OPT-Hybrid is to put a cache line into the sleep mode during $T_{i}$ if $T_{i}$ is greater than 1057, and to put it into the drowsy mode if $T_{i}$ falls into the range of $(6,1057]$. When $T_{i}$ is less than 6 , all the above methods keep the cache line active to ensure fast access time. When a sleep mode applied, the dynamic power consumption because of an induced miss was removed from the total leakage power savings.

Figure 10 depicts the percentage of leakage power in comparison with a cache with all its cache lines constantly active for each benchmark application. Figure 10a shows that for the instruction cache, the limit of leakage powersaving that OPT-Hybrid can achieve is $96.4 \%$. It is $26 \%$ higher than Sleep (10K), $16 \%$ higher than OPT-Sleep(10K), and 30\% higher than OPT-Drowsy. For the data cache (Figure 10b), the leakage power saving limit is $99.1 \%$, which is $15 \%$ higher than the Sleep(10K), 12\% higher than the OPT-Sleep(10K), and 33\% higher than the OPT-Drowsy. The results indicate that while the initial devised drowsy and sleep techniques are quite effective, there is still far more potential left in these techniques. Indeed, the leakage power savings for the optimal case are so large that it is fair to say that leakage power would become an insignificant portion of the total overall power if these savings could be realized. All these savings could be realized with new policies for cache management. Of course realizing these optimal numbers requires perfect knowledge of the address trace and timing, which is not typically possessed by a management policy.

\footnotetext{
${ }^{2}$ The sleep(10K) is similar to the cache-decay scheme in Kaxiras et al. [2001], in which the decay interval was set to $10 \mathrm{~K}$ cycles and the extra leakage power consumed by the counter per cache line was taken into account.
}

ACM Transactions on Architecture and Code Optimization, Vol. 2, No. 3, September 2005. 


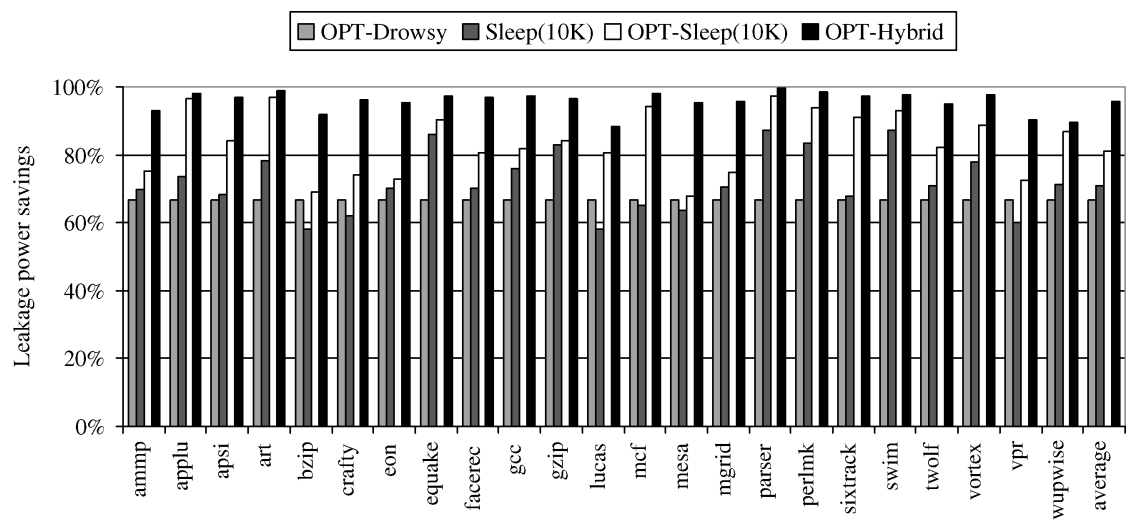

(a) Instruction Cache

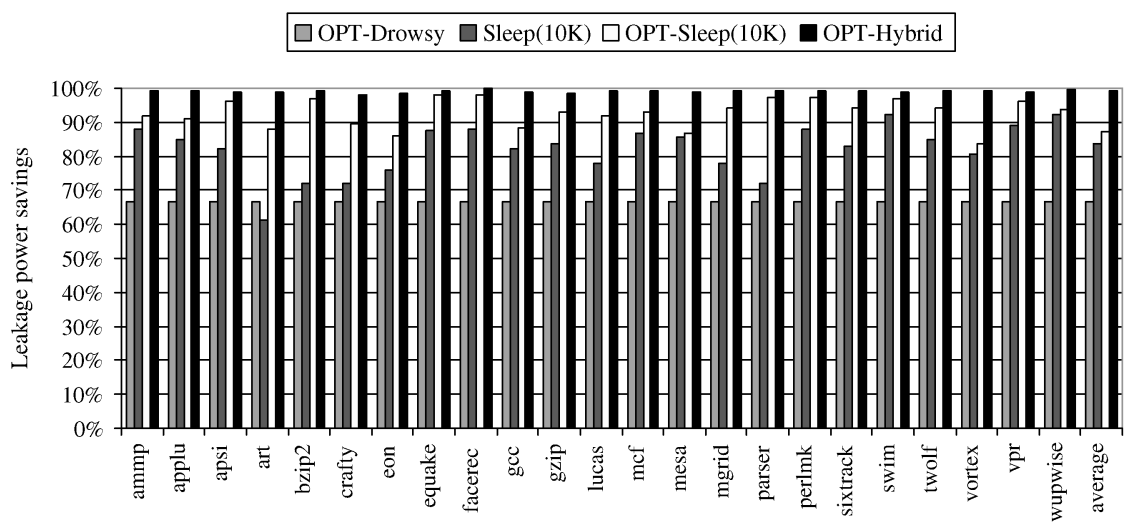

(b) Data Cache

Fig. 10. Comparisons of different leakage power-saving schemes.

\subsection{Limit Study for the Unified L2 Cache}

In modern processors, a large number of the total on-chip transistors is consumed by caches, particularly L2 caches. Different from L1 caches that are optimized for performance, L2 caches are optimized for density and stability, considering both yield and process parameter variation, and its memory cell size is usually smaller than a L1 SRAM cell. The area overhead of implementing either drowsy or sleep technique in L2 caches is about 5 to $8 \%$ and sleep mode may cause instability of L2 memory cells [Kim et al. 2004]. However, as machines and working-sets grow, the L2 caches are becoming increasingly performancecritical and integrated even closer to the processor (especially when a third level of hierarchy is added). In the future it is likely that L2 caches will be heavily accessed by multiple processors and will be close enough to the logic that they may well be affected by heat-dissipation problems. For example, currently, the L2 cache covers $37 \%$ of the alpha 21364 chip area and contains $85 \%$ of the total devices [Grodstein et al. 2002]. In addition, L2 caches have much larger miss penalty than L1 caches, because they have to go to main memory to fetch 


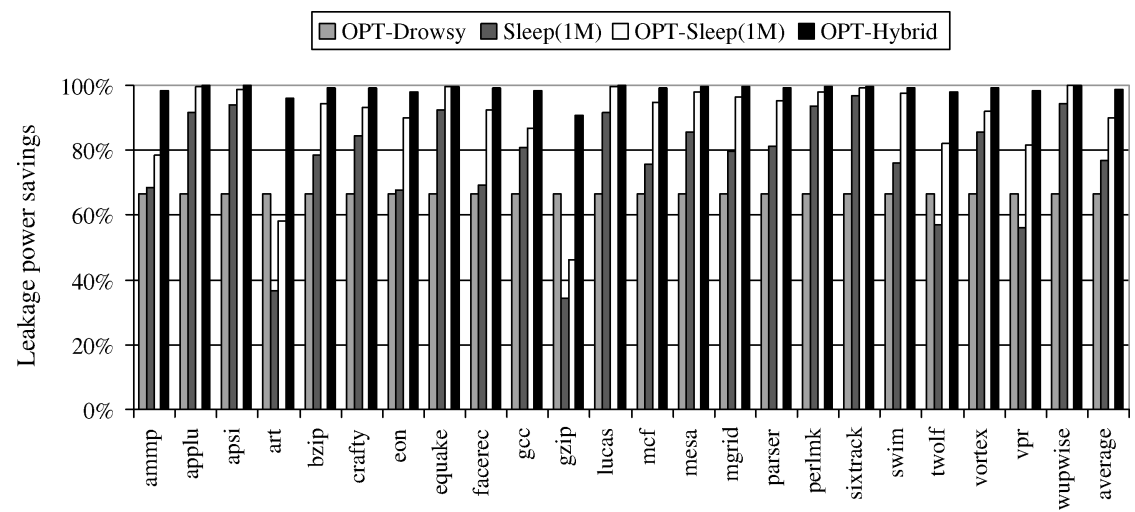

Fig. 11. Comparisons of different leakage power saving schemes for the direct-mapped L2 cache.

data when L2 misses occur. Thus, without careful attention to power, L2 caches may overwhelm the chip's power budget. In this section, we study the leakage reduction in L2 caches when both sleep and drowsy modes are employed.

For applications where the access frequency of L2 caches is small, using high-Vth transistors in memory cells will significantly reduce leakage power. Yet, for applications with large code and data footprints, more levels of cache hierarchy will be needed to take advantage of locality, e.g., the Intel Madison processor [Madison] has L2 caches which are frequently accessed. In those applications, solely applying high-Vth transistors in memory cells will reduce leakage, but may lead to significant performance impact, and dynamic management polices could be employed to trade off power and performance. In this work, we assume two level caches, while our optimal approach can be extended to study general multilevel caches. With the most advanced 70-nm implementation technology, we calculated the optimal sleep-drowsy inflection point for the L2 cache as 20760 cycles and the drowsy-active point as 6 cycles.

Figure 9c shows the average leakage power savings of all the benchmarks. It demonstrates how well the hybrid of sleep and drowsy modes can perform versus sleep mode. From the figure, we can see that if we can perfectly predict the access intervals of the L2 cache, there will be little benefit from using drowsy mode for those cache lines that are more frequently accessed than 20760 cycles. Figure 9c looks similar to Figure 9b, however, they are different in scale. The L2 cache has a much larger sleep-drowsy inflection point than the data cache (Figure $9 \mathrm{~b}$ ), since the miss penalty of the L2 cache is much larger than that of the on-chip data cache.

Figure 11 shows the comparison results of the four methods across all the benchmarks, OPT-Drowsy, OPT-Sleep (1M), Sleep $(1 M),{ }^{3}$ and OPT-Hybrid. The figure shows that for the L2 cache, the limit of leakage power saving that OPTHybrid can achieve is $97.7 \%$. It is $21 \%$ higher than Sleep(1M), $7.6 \%$ higher than

\footnotetext{
${ }^{3}$ The sleep(1M) is similar to the cache-decay scheme in Kaxiras et al. [2001], in which the decay interval was set to be $1 \mathrm{M}$ cycles, and the extra leakage power consumed by the counter per cache line was taken into account.
} 
Table II. Optimal Leakage Saving Percentages with Technology Scaling Down

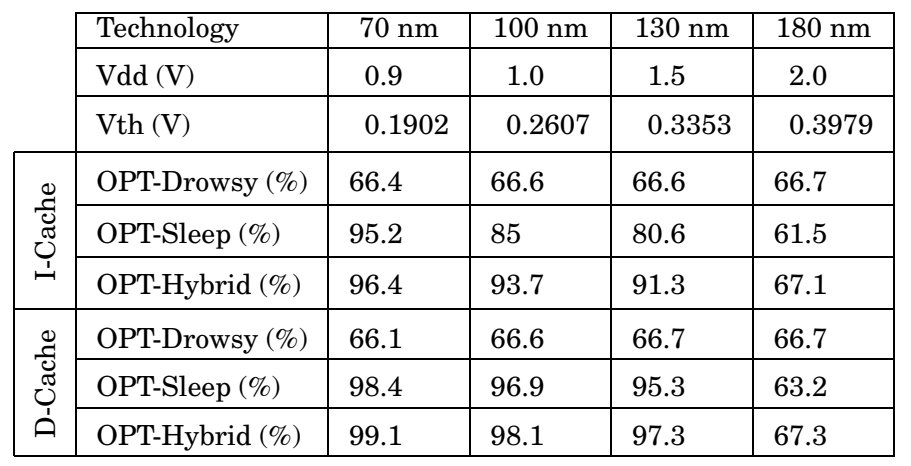

OPT-Sleep(1M), and 31\% higher than OPT-Drowsy, which indicates that there is still far more potential left in the existing techniques.

\subsection{Empirical Study with the Generalized Model}

We also evaluate the generality of the proposed parameterized model from two perspectives: one with different implementation technologies and the other with different cache configurations.

4.4.1 Evaluating the Generality of the Parameterized Model with Different Implementation Technologies. To show the generality of the parameterized model, we also study the L1 instruction and data caches with 100-, 130-, and 180 -nm processes. Table II summarizes the optimal leakage saving percentages we can possibly achieve by using OPT-Drowsy, OPT-Sleep, and OPT-Hybrid methods for each of these technologies. Instead of using OPT-Sleep(10K) on intervals that are greater than $10 \mathrm{~K}$ cycles, we study OPT-Sleep to determine what is the best leakage power saving we can achieve by aggressively turning off all intervals that are greater than the sleep-drowsy inflection point. The OPT-Drowsy and OPT-Hybrid methods are the same as before. The results in the table are the average results over all the benchmark applications.

The table illustrates that the leakage savings for both the instruction and the data caches of using OPT-Hybrid increase with the technology scaling down from 180 to $70 \mathrm{~nm}$. The increment of the possible leakage savings is due to the decrement of the sleep-drowsy inflection point. Moreover, the table shows that for the 180-nm technology implementation, the drowsy mode plays a more important role in saving leakage power than the sleep mode does; for the others, the sleep mode plays a significant role. This can be also attributed to the large difference of the sleep-drowsy inflection points. Finally, the table also reveals that more leakage savings can be possibly achieved with the technology scaling down, which leaves us more space for further improvement on leakage power savings.

4.4.2 Evaluating the Generality of the Parameterized Model with Different Cache Configurations. To further evaluate the generality of the parameterized model on studying the limits of leakage power reduction, we also conducted 


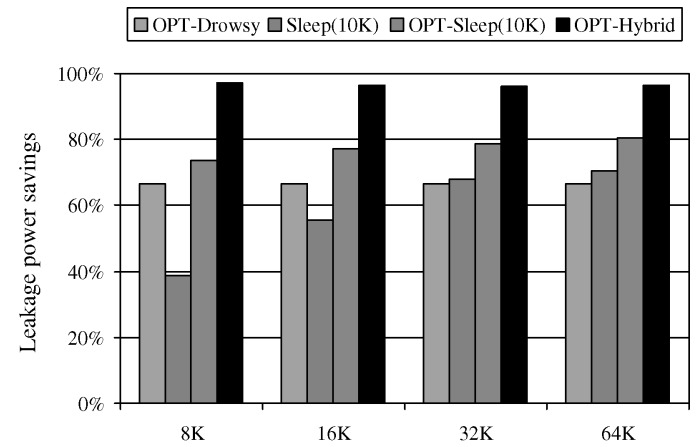

(a) L1 Instruction Caches

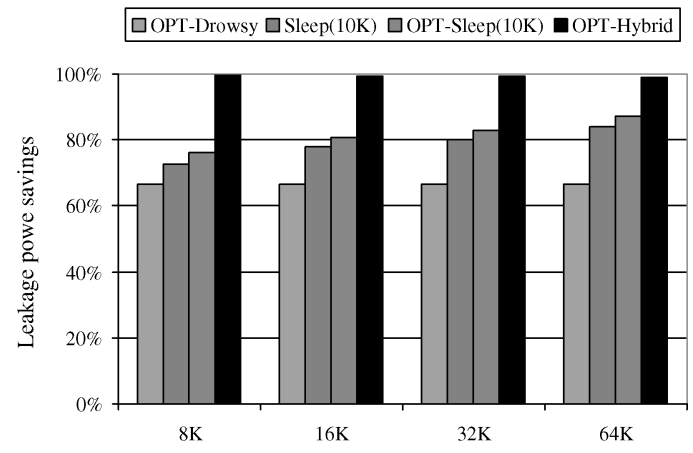

(b) L1 Data Cache

Fig. 12. Comparison of different leakage power saving schemes for $\mathrm{L} 1$ caches with different sizes.

experiments on L1 caches with different sizes, while the remainder of the configurations remain the same. Specifically, we studied 8-, 16-, and 32-KB two-way set associative instruction and data caches with one-cycle latency. Figure 12 shows results, on average, of different L1 caches. They demonstrate that as the L1 cache sizes get larger, the percentage of the leakage power saving for each scheme (OPT-Drowsy, Sleep(10K), OPT-Sleep(10K), and OPT-Hybrid) increases. The drowsy mode plays a more important role for smaller caches than/does the sleep mode. This is because using smaller caches usually results in more frequent cache misses, which lead to many small intervals (less than $10 \mathrm{~K}$ cycles) because of frequent replacements. Because of the existence of the small intervals, the drowsy mode shares a significant portion in reducing leakage power.

In addition to studying the leakage problem with different $\mathrm{L} 1$ cache sizes, we also experimented with another configuration that has a different L2 cache configuration. This configuration includes a $32-\mathrm{KB}$ two-way set associative instruction cache with a single-cycle hit latency, a 32-KB two-way set associative data cache with a two-cycle hit latency, and a unified 1-M two-way set associative L2 cache with five-cycle hit latency. The rest of the configuration, such as the main memory, replacement policy, and implementation technology, are the same as the previous configurations. 


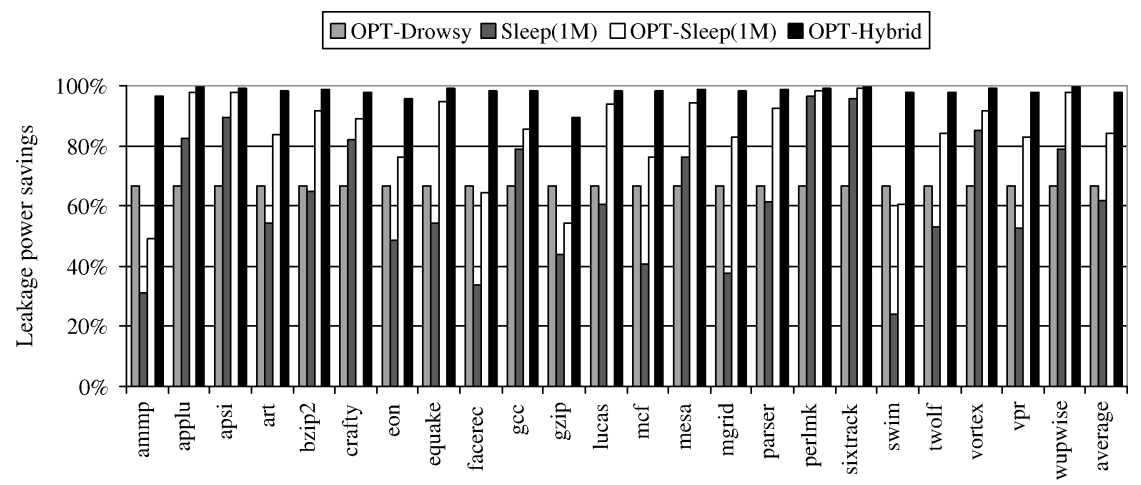

Fig. 13. Comparisons of different leakage power-saving schemes for the new two-way associative L2 cache.

Figure 13 shows the comparison results of the smaller L2 cache for different leakage power-saving techniques. For the purpose of comparison with the previous cache configuration, the results here also include the results of all benchmarks and their average. Note that due to the different cache configurations, the baseline of the total leakage power consumption of the new configuration (Figure 13) is different from that of the previous configuration (Figure 10), although the $y$-axis also indicates the percentage of total leakage power of which all lines remain constantly active. In our experiments, we found that the total power averaged over all benchmarks for the previous configuration was twice that of the new configuration.

From Figures 10 and 13, we can make three observations. First, for OPTD,rowsy, Sleep $(1 \mathrm{M})$, and OPT-Sleep $(1 \mathrm{M})$, there are still much room left for further exploring circuit and architectural techniques to achieve the maximal leakage power savings that OPT-Hybrid provides. Second, the percentages of leakage power savings for Sleep $(1 \mathrm{M})$ and OPT-Sleep(1M) on the new L2 cache are smaller than those on the previous L2 cache. Third, the results of ammp and $m c f$ clearly show that OPT-Drowsy achieves more leakage power savings than Sleep (1M) and OPT-Sleep(1M) with the new configuration, while OPTDrowsy saves less leakage power than either Sleep(1M) or OPT-Sleep(1M). The explanation for the above observations is that the new cache configuration has smaller L1 caches than the previous configuration, which will generally result in a larger number of accesses to the L2 cache and smaller access intervals for the L2 cache lines. Since most of the power savings are from the long intervals, the new cache configuration consequently only achieves less power savings than the previous configuration. This confirms that the parameterized model can be generalized to provide limits of leakage power reduction for different cache configurations.

We would also like to mention that we had an initial exploration [Meng et al. 2005] of using a form of prefetching, such as next-line and stride-based techniques, to approximate the optimal. The goal of prefetching is to accurately predict future access patterns so that they can optimistically fetch from memory before their use. We propose that prefetching can optimistically refetch data 
that has been either turned off for sleep mode or put into a drowsy state. In our trial study, we delivered the information of what is the best those employed prefetching techniques can help in approaching the optimal and we, thus, did not take into consideration the overhead of prefetching. Our evaluation of the potential usefulness of next-line and stride-based prefetching toward reducing leakage power on the L1 instruction and data caches shows that if prefetching can be used to guide sleep mode and drowsy mode is used the other times, then the leakage power dissipation will be within a factor of 2.5 from the optimal.

\section{CONCLUSIONS}

Leakage power dissipation is quickly becoming a major concern in designing high-performance processors. In this paper, we explore the limits to which known circuit-level techniques can be combined and employed to save cache leakage power using new management methods and protocols. In addition, we developed a parameterized model to determine the optimal leakage savings while the implementation technology changes over time. We find that it is possible with perfect knowledge of the future address trace to reduce the amount of power dissipated by the instruction cache down by a factor of 5.3 from known techniques ( 2 for the data cache, and 10 for the unified L2 cache). At this level, the leakage power of the cache would become a less serious problem. Through the evaluation of generality of the parameterized model, we found the model is robustly applicable to different caches, which will provide helpful guidance for further researches in cache-level leakage power reduction.

\section{ACKNOWLEDGMENTS}

The authors would like to thank reviewers for their valuable feedback on the manuscript.

\section{REFERENCES}

Agarwal, A., Li, H., AND Roy, K. 2002. DRG-cache: a data retention gated-ground cache for low power. In Proceedings of Design Automation Conference.

Amrutur, B. S. ANd Horowitz, M. A. 2001. Fast low-power decoders for RAMs. IEEE Journal of Solid-State Circuits 36, 10, 1506-1515.

Azizi, N., NAJM, F. N., AND Moshovos, A. 2003. Low-leakage asymmetric-cell SRAM. IEEE Transactions on Very Large Scale Integration Systems 11, 4 (Aug.).

BAI, R., Kim, N. S., Mudge, T., AND Sylvester, D. 2005. Power-performance trade-offs in nanometerscale multi-level caches considering total leakage. In Proceedings of Design, Automation and Test in Europe.

BELADY, L. 1966. A study of replacement of algorithms for a virtual storage computer. IBM Systems Journal 5, 2, 78-101.

Desikan, R., Burger, D., Keckler, S. W., and Austin, T. M. 2001. Sim-alpha: a validated execution driven alpha 21264 simulator. Tech. Rep. TR-01-23, Department of Computer Sciences, University of Texas at Austin.

Flautner, K., Kim, N., Martin, S., Blaauw, D., and Mudge, T. $2002 . \quad$ Drowsy caches: simple techniques for reducting leakage power. In Proceedings of International Symposium on Computer Architecture. Anchorage, AK.

Grodstein, J., Gowan, M., Lane, R., Lilly, B., Nagalla, K., Shah, R., Shriver, E., Yin, S., Morton, S., Rayess, R., Truex, T., Shattuck, L., Lowell, S., Bailey, D., Bertucci, D., Bischoff, G., And 
Dever, D. 2002. Power and cad considerations for the 1.75 mbyte, $1.2 \mathrm{ghz} 12$ cache on the alpha $21364 \mathrm{cpu}$. In Proceedings of the 12th ACM Great Lakes symposium on VLSI. New York, NY.

Hanson, H., Agarwal, Hrishikesh, M., Keckler, S., and Burger, D. 2001. Static energy reduction techniques for microprocessor caches. In Proceedings of International Conference on Computer Design.

Heo, S., Barr, K., Hampton, M., And Asanovic, K. 2002. Dynamic fine-grain leakage reduction using leakage-biased bitlines. In Proceedings of International Symposium on Computer Architecture. Anchorage, Alaska.

H. KIm AND Roy, K. 2002. Dynamic vt SRAM's for low leakage. In Proceedings of ACM International Symposium on Low Power Design.

Hu, J. S., NADGiR, A., VijaYkrishnan, N., Irwin, M. J., AND KandemiR, M. 2003. Exploiting program hotspots and code sequentiality for instruction cache leakage management. In Proceedings of International Symposium on Low Power Electronics and Design. Seoul, Korea.

Hu, Z., KaXiras, S., AND Martonosi, M. 2002. Let caches decay: Reducing leakage energy via exploitation of cache generational behavior. ACM Transaction on Computer Systems.

KAXIRAS, S., Hu, Z., AND MARTONOSI, M. 2001. Cache decay: exploiting generational behavior to reduce cache leakage power. In Proceedings of International Symposium on Computer Architecture. Göteborg, Sweden.

KESSLER, R. 1999. The alpha 21264 microprocessor'. In Proceedings of IEEE Micro. 24-36.

Kim, N., Flautner, K., Blaauw, D., and Mudge, T. 2002. Drowsy instruction cache: leakage power reduction using dynamic voltage scaling and cache sub-bank prediction. In Proceedings of ACM / IEEE International Symposium on Microarchitecture. Istanbul, Turkey.

Kim, N., Flautner, K., BlaAuw, D., And Mudge, T. 2004. Circuit and microarchitectural techniques for reducing cache leakage power. IEEE Transaction on Very Large Scale Integration Systems 12, 2 (Feb.), 167-184.

Kim, N. AND Mudge, T. 2004. Single vdd and single vt super-drowsy techniques for low-leakage high-performance instruction caches. In Proceedings of International Symposium on Low Power Electronics and Design. Newport Beach, CA.

Kim, N. S., Austin, T., Blaauw, D., Mudge, T., Flautner, K., Hu, J., Irwin, M., Kandemi, M., and VIJAYKRISHNAN, N. 2003. Leakage current: Moore's law meets static power. Computer 36, 12 (Dec.).

Lee, D., Blaauw, D., And Sylvester, D. 2004. Gate oxide leakage current analysis and reduction for VLSI circuits. IEEE Transactions on Very Large Scale Integration Systems 12, 2 (Feb.).

Li, L., Kadayif, I., Tsai, Y., ViJaykrishnan, N., Kandemir, M., Irwin, M. J., and Sivasubramaniam, A. 2003. Managing leakage energy in cache hierarchies. Journal of Instruction-level Parallelism 5.

Li, Y., Parikh, D., Zhang, Y., Sankaranarayanan, K., Skadron, K., and Stan, M. 2004. Statepreserving vs. non-state-preserving leakage control in caches. In Proceedings of the 2004 Design, Automation and Test in Europe Conference.

Liu, D. And Svensson, C. 1993. Trading speed for low power by choice of supply and threshold voltages. IEEE Journal of Solid State Circuits 28, 1 (Jan.).

LiU, J. AND ChoU, P. 2004. Optimizing mode transition sequences in idle intervals for componentlevel and system-level energy minimization. In Proceedings of International Conference on Computer Aided Design. San Jose, CA.

Meng, Y., Sherwood, T., AND Kastner, R. 2005. On the limits of leakage power reduction in caches. In Proceedings of International Symposium on High-Performance Computer Architecture. San Francisco, CA.

Powell, M., Yang, S., Falsafi, B., Roy, K., and ViJaykumar, T. N. 2001. Reducing leakage in a high-performance deep-submicron instruction cache. IEEE Transactions on Very Large Scale Integration Systems 9, 1 (Feb.).

Rabaey, J., Chandrakasan, A., And Nikolic, B. 2002. Digital Integrated Circuits A Design Perspective(2nd Edition). Prentice-Hall.

SAmSUng Electronics. 2002. DDR2SDRAM datasheet MR16R1622.

Sankaranarayanan, K. and Skadron, K. 2004. Profile-based adaptation for cache decay. ACM Transactions on Architecture and Code Optimization 1, 3 (Sep.). 
Sherwood, T., Perelman, E., Hamerly, G., and Calder, B. 2002. Automatically characterizing large scale program behavior. In Proceedings of International Conference on Architectural Support for Programming Languages and Operating Systems. San Jose, CA.

Shivakumar, P. AND Jouppi, N. 2001. CACTI 3.0: An integrated cache timing, power, and area model. Tech. Rep. WRL-2001-2, HP Labs Technical Reports. Dec.

Velusamy, S., Sankaranarayanan, K., Parikh, D., Abdelzaher, T., and Skadron, K. 2002 . Adaptive cache decay using formal feedback control. In Proceedings of 2002 Workshop on Memory Performance Issues in conjunction with ISCA-29. Anchorage, Alaska.

Zhang, W., Hu, J. S., Degalahal, V., Kandemir, M., Vijaykrishnan, N., and Irwin, M. J. 2002. Complier-directed instruction cache leakage optimization. In Proceedings of International Symposium on Microarchitecture. Istanbul, Turkey.

Zhang, Y., Parikh, D., Sankaranarayanan, K., Skadron, K., and Stan, M. R. 2003. Hotleakage: An architectural, temperature-aware model of subthreshold and gate leakage. Tech. Rep. Tech. Report CS-2003-05, Department of Computer Sciences, University of Virginia. Mar.

Zhou, H., Toburen, M. C., Rotenberg, E., And Conte, T. M. 2003. Adaptive mode control: a static power-efficient cache design. ACM Transcactions on Embedded Computing Systems 2, 3 (Aug.).

Received January 2005; revised May 2005; accepted July 2005

ACM Transactions on Architecture and Code Optimization, Vol. 2, No. 3, September 2005. 